\title{
Prediction of Inhomogeneous Stress in Metal Structures: A Hybrid Approach Combining Eddy Current Technique and Finite Element Method
}

\author{
Yating Yu $\mathbb{D}^{1,2}$ Fei Yuan $\mathbb{D}^{1},{ }^{1}$ Hanchao Li $\mathbb{D}^{1},{ }^{1}$ Cristian Ulianov $\mathbb{D}^{3},{ }^{3}$ and Guiyun Tian $\mathbb{D}^{3}$ \\ ${ }^{1}$ School of Mechanical and Electrical Engineering, University of Electronic Science and Technology of China, 611731 Chengdu, China \\ ${ }^{2}$ Institute of Electronic and Information Engineering of UESTC in Guangdong, 523808 Dongguan, China \\ ${ }^{3}$ School of Engineering, Newcastle University, Newcastle upon Tyne NE1 7RU, UK
}

Correspondence should be addressed to Yating Yu; yuyating-uestc@hotmail.com

Received 25 October 2020; Accepted 15 June 2021; Published 7 July 2021

Academic Editor: Fanli Meng

Copyright (c) 2021 Yating Yu et al. This is an open access article distributed under the Creative Commons Attribution License, which permits unrestricted use, distribution, and reproduction in any medium, provided the original work is properly cited.

\begin{abstract}
Concentrated stresses and residual ones are critical for the metal structures' health, because they can cause microcracks that require emergency maintenance or can result in potential accidents. Therefore, an accurate approach to the measurement of stresses is key for ensuring the health of metal structures. The eddy current technique is an effective approach to detect the stress according to the piezoresistive effect. However, it is limited to detect the surface stress due to the skin effect. In engineering, the stress distribution is inhomogeneous; therefore, to predict the inhomogeneous stress distribution, this paper proposes a nondestructive approach which combines the eddy current technique and finite element (FE) method. The experimental data achieved through the eddy current technique determines the relationship between the applied force and the magnetic flux density, while numerical simulations through the FE method bridge the relationship between the magnetic flux density and the stress distribution in different directions. Therefore, we can predict the inhomogeneous stress nondestructively. As a case study, the applied stress in a threepoint-bending simply supported beam was evaluated, and the relative error is less than $8 \%$ in the whole beam. This approach can be expected to predict the residual stress in metal structures, such as rail and vehicle structures, if the stress distribution pattern is known.
\end{abstract}

\section{Introduction}

Stress concentration, or residual stress, is the main cause of the micro cracks in metal components and structures (such as oil/gas pipeline [1,2], airfoil [3], steel bridge [4], hoisting equipment [5], and polycrystalline solids [6]). Consequently, the microcracks in such key components and subassemblies can suddenly cause structure failure when operating under alternating loads; this may lead to economic and environmental losses, as well as human casualties $[7,8]$, if there is no appropriate maintenance strategy. Therefore, the evaluation approach to the stress distribution is essential to control the early stage quality and to extend the lifetime by structural health monitoring. However, it is typically difficult to measure or predict the residual stress [9].
Non-Destructive Testing (NDT) techniques play a vital role in assuring safety and serviceability of a variety of key infrastructure assets and facilities [10-24]. The commonly applied techniques in stress or residual stress measurement are X-ray diffraction, ultrasonic testing, magnetic memory method (MMM), magnetic Barkhausen noise (MBN), and eddy current testing (ECT) [11-19]. The X-ray diffraction technique is suitable for the surface stress measurement [6]; however, it is not efficient for measuring the stress in depth. In engineering, it is usually combined with other destructive techniques to extend the measurement depth to overcome this problem [11]. The ultrasonic technique has a higher measurement depth than the X-ray diffraction technique; however, it has high requirements on the material surface and its measurement accuracy is low [12]. MMM utilizes 
the geomagnetic field and magnetostrictive effect to detect the stress or residual stress; therefore, it is suitable for the online and the real-time measurement, but its detection signal is too weak to easily be interfered by the environment noise, so the detection accuracy is not very high $[13,14]$. Barkhausen noise can measure the surface and subsurface stress but is limited to magnetic material, because the Barkhausen effect only exists in the ferrite material $[15,16]$. In addition to this, for the crack bridging stress, Greene et al. adopted the Raman microprobe technique to measure bridging stresses for fatigue samples. However, the calibration is necessary because the Raman shift can be affected by changes in chemical composition [17]. The eddy current technique has a low cost and low sensitivity to environmental influence (e.g., moisture and dust) and is suitable for quantitative surface/subsurface measurements (e.g., crack detection [18-21], magnetic permeability and electrical conductivity characterization [22], and displacement measurement [23]) for the conductive material. Due to the piezoresistive effect of conductive material, the eddy current technique appears to be more advantageous over other techniques for stress evaluation in metal structures. Some investigations [24] indicated that the eddy current response is sensitive to stress changes in metals.

Therefore, more and more research works for stress measurement are focused on the use of the eddy current technique. In 2001, Ricken et al. employed a Giant Magneto Resistive (GMR) sensor and an eddy current sensor to characterize the axial stress of steel wire. The investigations indicate that the magnetic flux density detected by the GMR sensor is related to the applied load, while the resistance and the inductance of the eddy current sensor are a function of the mechanical stress [25]. However, the plasticity $[24,25]$ and aging/heat treatment [26] also affect the electrical conductivity of metals as well as applied stress. Therefore, they have a combined influence on the eddy current response. Morozov and Tian [27] deeply investigated the eddy current response of samples of aluminum alloys (AA-1050, AA-2024, AA-5083, and AA-7075) with different levels of plastic deformation and different heat treatments. The aluminum alloys subjected to elastic uniaxial loading were monitored by the circular and directional eddy current probes. The experimental results indicated that the stress coefficients are generally positive and depend on the annealing (heat treatment) condition as well as the level of prior plastic work; the rectangular probe is much more sensitive when oriented normally to the tensile stress. After that, they continued to study the response of the eddy current on the change of electrical conductivity due to the elastic and plastic stress. They pointed out that the real part of the EC response is sensitive mainly to elastic stress, while the imaginary part of the EC response is sensitive mainly to plastic stress [28]. Zhou et al. [29] proposed a pulse electromagnetic method (PEM) with a U-shaped sensor to detect the unidirectional tension stress in ferromagnetic metals.

The existing literatures illustrate that the eddy current technique is an effective nondestructive technique for measuring stress. The stress distribution is generally inhomogeneous under the complex load in manufacturing and in service. However, only limited literatures considered the inhomogeneous stress. Nagy et al. [30-32] proposed the residual stress assessment for nickel-based superalloys after the shot-peened processing by the eddy current technique. While their investigation also discovered that the relationship between the electrical conductivity profile and the sought residual stress profile is very sensitive to the sample's state of precipitation hardening [33] and thermoplastic effect [34]. Furthermore, the eddy current can detect the surface stress due to the skin effect. Therefore, the prediction accuracy for the inhomogeneous stress should be improved further by combining other methods. Ahn et al. [35] combine the X-ray diffraction and FEM together to the prediction of welding residual stresses in fibre laser-welded AA2024-T3, while Zhu et al. [36] proposed an approach to determine the residual stress in metal by combining slot milling method and finite element approach, and the determination result by the proposed method is verified by the X-ray diffraction. However, the slot milling method is a destructive method while X-ray diffraction is expensive and is harmful for the operator's health.

Therefore, a prediction approach is proposed by combination the eddy current technique and finite element method (FEM) to evaluate the inhomogeneous distribution stress in metals accurately and nondestructively. The paper is structured as follows: the fundamental theories for the stress evaluation by the eddy current technique are presented in Section 2; the inhomogeneous stress distribution evaluation approach based on the eddy current technique and FE numerical simulation is proposed in Section 3; a case study, i.e., measurement of stress distribution in a simply supported beam with a three-point-bending deformation, is shown in Section 4. Section 5 draws conclusions and makes recommendations for future work.

\section{Fundamental Theory}

2.1. Piezoresistive Effect. The geometry and resistivity of metals change when mechanical loads are applied and, consequently, cause the change of the metal's resistance. The change of material's resistivity due to applied loads is called the piezoresistive effect. Considering the cuboid metal as an example, its resistance can be expressed as

$$
R=\frac{\rho \cdot l}{w \cdot t h},
$$

where $\rho$ is the resistivity of the metal $(\Omega \mathrm{m}), l$ is the length of the cuboid (mm), $w$ is the width of the cuboid ( $\mathrm{mm}$ ), and th is the thickness of the cuboid (mm).

According to the piezoresistive effect, if the metal is stretched in length, $\rho, l, w$, and th are all changed. The change of the resistance of cuboid metal due to the changes of $\rho, l, w$, and th can be expressed as

$$
\frac{\mathrm{d} R}{R}=\frac{\mathrm{d} l}{l}+\frac{\mathrm{d} \rho}{\rho}-\frac{\mathrm{d} w}{w}-\frac{\mathrm{d} t h}{t h} .
$$

Assuming that the strain along the length is $\varepsilon$, which 
equals to $d l / l$, the strain in width and thickness can be expressed as

$$
\left\{\begin{array}{l}
\frac{\mathrm{d} w}{w}=-v \mathcal{E} \\
\frac{\mathrm{dth}}{\mathrm{th}}=-v \mathcal{E}
\end{array}\right.
$$

where $v$ is the Poisson's ratio of the metal.

Therefore, Equation (2) can be simplified to

$$
\frac{\mathrm{d} R}{R}=(1+2 v) \cdot \varepsilon+\frac{\mathrm{d} \rho}{\rho},
$$

where $(1+2 v) . \varepsilon$ is the change in resistance due to the change of the geometry of the cuboid and $d \rho / \rho$ is the change in resistance due to the piezoresistive effect.

Therefore, the resistance change in metals stems from the change of their geometry and the change of the resistivity resulting from the applied mechanical stress. For some metals, such as platinum alloy, the resistance change due to piezoresistivity is much larger than that due to geometry change.

\subsection{Operation Principle of Eddy Current Method for Stress} Measurement. The operation principle of the eddy current testing instrument is shown in Figure 1. An alternating current $I$ in the driving coil creates an alternating magnetic field $H_{1}$, which is the primary magnetic field and induces current $I_{2}$ in the sample. The eddy currents simultaneously generate a secondary magnetic field $\mathrm{H}_{2}$, which resists the variation of the primary magnetic field and changes the resultant magnetic field $H$.

The geometric parameters of coil, such as the number of turns $N$, the inner radius $r_{1}$, the outer radius $r_{2}$, and the height $h_{c}$, are key factors to the primary magnetic field $H_{1}$; the lift-off $l$, sample's electrical conductivity $\sigma_{\text {ele, }}$, and sample's relative magnetic permeability $\mu_{r}$ affect the secondary magnetic field $\mathrm{H}_{2}$; the excitation current $I$ and the excitation angular frequency $\omega$ have influence on the primary magnetic field $H_{1}$ as well as on the secondary magnetic field $H_{2}$.

Therefore, $H$ is dependent on factors such as the lift-off, excitation frequency, sample electrical conductivity, sample relative magnetic permeability, and probe coil geometry. The $Z$-component of magnetic flux density $B_{z}$ is commonly used as a detection signal due to its detectability and strength. Therefore, $B_{z}$ can be expressed as Equation (5)

$$
B_{z} \sim B_{z}\left(N, I, r_{1}, r_{2}, l, h_{c}, \sigma_{e l e}, \mu_{r}, \omega\right) .
$$

According to Equations (4) and (5), the applied mechanical stress can cause the change of electrical conductivity of the sample due to geometry change and piezoresistivity effect. Consequently, the change of sample's electrical conductivity can induce the change of resultant magnetic flux density. Therefore, the eddy current technique can theoretically reflect the applied stress in metals.

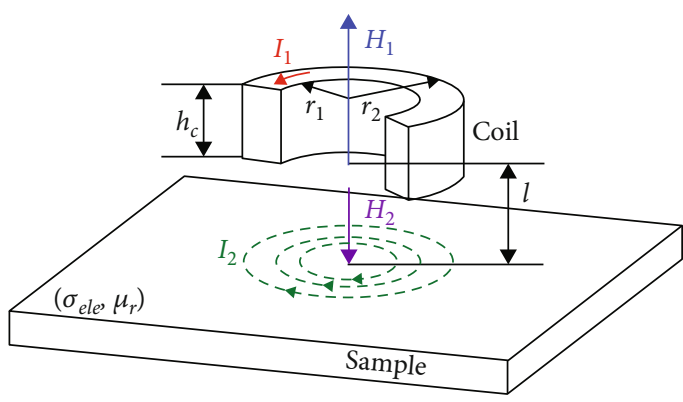

FIgURE 1: The operation principle of the eddy current technique.

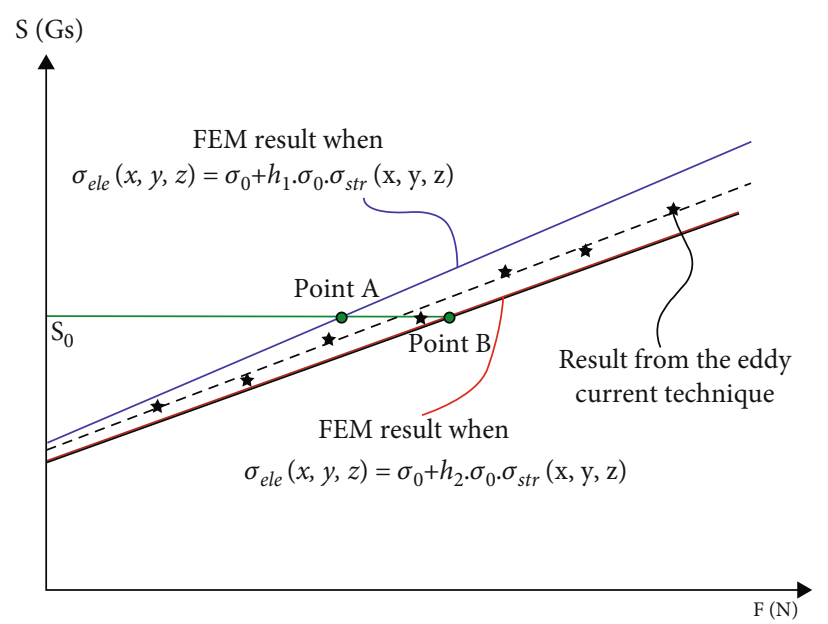

FIGURE 2: Schematic graph for the approach to measuring of inhomogeneous stress.

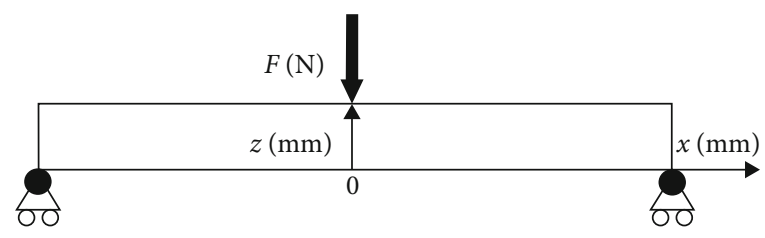

FIgURE 3: Three-point-bending simply supported beam.

The actual size of the sample is much larger than the distribution range of the magnetic field in the eddy current method, so the stress detected by the eddy current technique can reflect the piezoresistivity effect accurately in metals.

\section{Hybrid Approach for Inhomogeneous Distribution Stress}

Ricken et al. [25] investigated the relationship of between the resistance and inductance of the coil of the eddy current sensor and the stress, and the results indicated that the impedance of the coil is linear changing with the stress. Therefore, the linear relationship between the eddy current detection signal $S$ and the applied force $F$ is assumed as Equation (6), which can be symbolically presented as the dotted line in Figure 2.

In Figure $2, \star$ represents symbolically the data from the experiment by the eddy current technique. The coefficient 


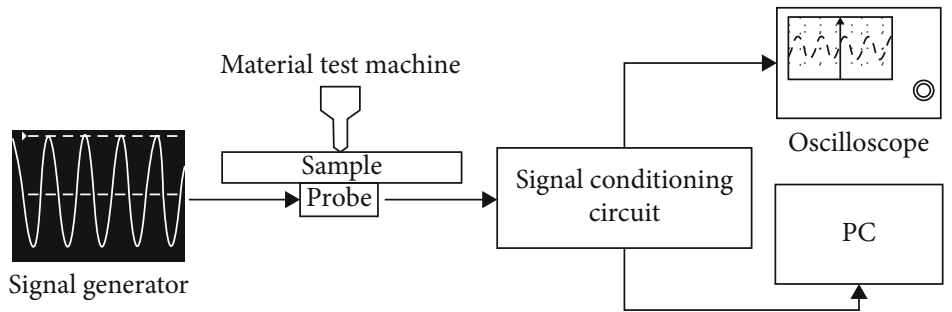

Figure 4: Schematic diagram of the experiment setup.

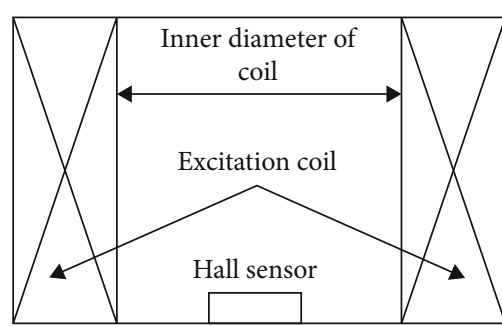

(a)

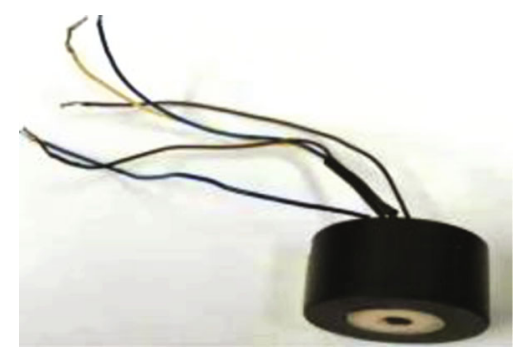

(b)

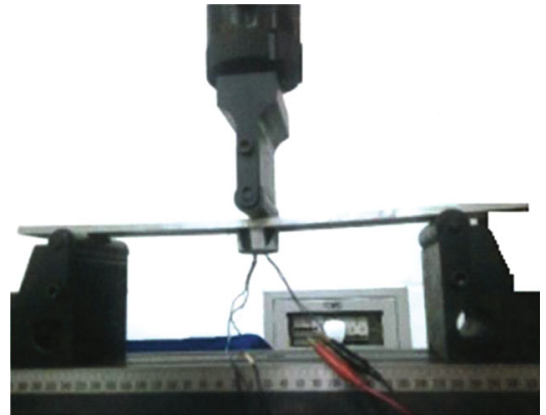

(c)

Figure 5: The probe (a) the structural diagram of probe, (b) the real probe, and (c) the location of the probe in the experiment.

of determination of the dotted line with the experiment data is denoted as $R_{s 0}$.

$$
S=K F+a,
$$

where $S$ is the detection signal of the eddy current technique, $F$ is the applied force, and $K$ and $a$ are the slope and the intercept of the linear relationship between $S$ and $F$, respectively, which can be determined by experiment.

According to the mechanics of material, when the force $F$ is applied on the conductive sample, the generated internal force can be calculated as $G(F)$, and the stress distribution on the cross-section of the conductive sample can be generalized as

$$
\sigma_{\text {str }}(x, y, z)=\frac{G(F) \cdot C(x, y, z)}{P_{\text {geom }}(w, l, t h)}
$$

where $C(x, y, z)$ are the coordinates of the detection point in $x$-axis, in $y$-axis, and in $z$-axis; $P_{\text {geom }}(w, l$, th $)$ is constant when the geometry of the sample is determined; $w$, th is width and height of the sample cross-section, and $l$ is the length of the sample.

Gong et al. [37] found that the change of resistivity of the conductive sample is linear with the change of strain of the sample. According to the constitutive relationship of stress and strain, the electrical conductivity in the cross-section $\sigma_{\text {ele }}(x, y, z)$ can be assumed as

$$
\sigma_{\text {ele }}(x, y, z)=\sigma_{0}+h \cdot \sigma_{0} \cdot \sigma_{\text {str }}(x, y, z),
$$

where $\sigma_{0}$ is the electrical conductivity of the sample without
TABLE 1: The influence of the applied force on the signal from the hall sensor.

\begin{tabular}{lcc}
\hline Applied force $(\mathrm{N})$ & Voltage from the hall sensor $(\mathrm{mV})$ & $B_{z}(\mathrm{Gs})$ \\
\hline 40 & 4080.828 & 70.58 \\
80 & 4085.593 & 71.59 \\
120 & 4090.988 & 72.74 \\
160 & 4093.198 & 73.22 \\
200 & 4096.094 & 73.83 \\
240 & 4102.996 & 75.31 \\
\hline
\end{tabular}

stress and $h$ is a conversion coefficient between the stress and electrical conductivity of the sample.

As shown in Figure 2, the range of coefficient $h$ is $h_{2}$ $<h<h_{1}$, where $h_{1}$ is the upper limit and $h_{2}$ is the lower limit. The coefficient $h_{2}$ can be obtained according to the following steps:

Step 1. $n=1$.

Step 2. $h=n \times h_{0}$, where $h_{0}$ is a tiny positive value.

Step 3. Set the conductivity distribution in the numerical model according to Equation (8).

Step 4. Obtain the relationship of $F \sim S$, and calculate its coefficient of determination $R_{s}$.

Step 5. If $\left|R_{s}-R_{s 0}\right|<\lambda$ ( $\lambda$ is a tiny positive value), we denote the $h_{2}=h$; if no, set $n$ as $n+1$, and turn to Step 2 . 


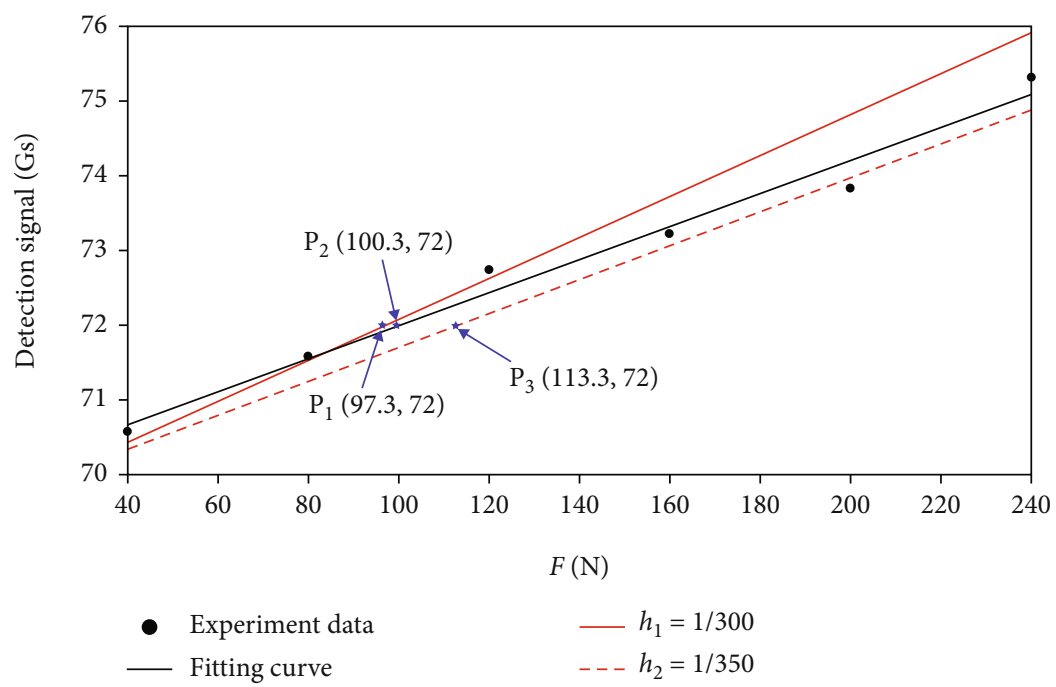

Figure 6: Prediction of the coefficient $h$ by experimental study.

In order to get the upper limit $h_{1}$, we can represent $h=n \times h_{0}$ in step 2 as $h=h_{1}+n \times h_{0}$, as well as represent $h_{2}=h$ in step 5 as $h_{1}=h$. Therefore, the range of coefficient $h$ can be determined as $h_{2}<h<h_{1}$.

As shown in Figure 2, if we know the detection signal is $S_{0}$ , we can get the point $\mathrm{A}$ and point $\mathrm{B}$. Point $\mathrm{A}$ is the intersection point of $S_{0}$ and the line $h=h_{1}$ while Point B is the intersection point of $S_{0}$ and line $h=h_{2}$. Therefore, we can get the applied forces for point $A$ and point $B$, and we denote the applied force for point $\mathrm{A}$ is $F_{1}$ and denote that for point $\mathrm{B}$ is $F_{2}$. After that, we obtained the applied force $F_{m}$ as the average value of $F_{1}$ and $F_{2}$. Finally, we can get the stress distribution according to Equation (7).

As illustrated above, in Step 3, we input the conductivity distribution in the numerical model according to Equation (8) and can get the detection signals under different applied loads. Then, in Step 4 we can obtain the relationship of $F \sim$ $S$ and calculate the coefficient of determination $R_{s}$ between the $F \sim S$ and experimental data from the eddy current technique. Therefore, the electromagnetic data from the eddy current technique and the mechanical model by numerical simulation are combined.

\section{Case Study}

In the case study, the continuous and inhomogeneous stress distribution in a simply supported beam for three-pointbending deformation is considered. The beam material is aluminum alloy 7075 and its size is $250 \mathrm{~mm} \times 35 \mathrm{~mm} \times 6$ $\mathrm{mm}$. The sketch of three-point-bending experiment is shown in Figure 3.

\subsection{Experimental Investigation on Relationship of $F \sim B_{z}$}

4.1.1. Experimental Setup. The experimental setup (shown in Figure 4) includes the power supply for the eddy current probe, the signal generator, the signal conditioning circuit, aluminum alloy 7075, signal acquisition system, and material test machine (SANS CM75105). The probe consists of the

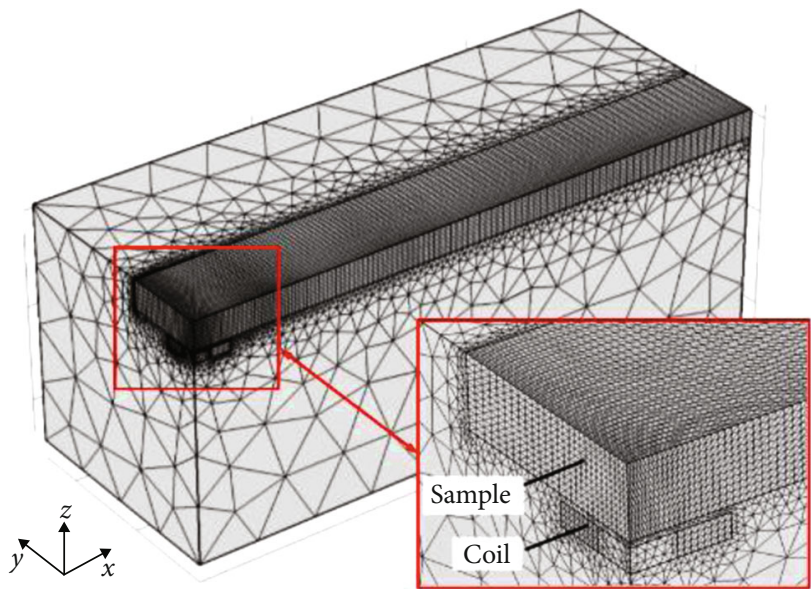

FIGURE 7: The FE model.

excitation coil and the hall sensor SS495A. The Hall sensor SS495A is a linear magnetic sensor, which can reflect $B_{z}$ by the output voltage signal linearly. The structural diagram of the probe and the real probe is shown in Figure 5. In the experiment, the probe is located below the applied force, as shown in Figure $5(\mathrm{c})$, and a $10 \mathrm{~V}$ voltage signal of $5 \mathrm{kHz}$ is input the probe as the excitation signal. The impedance of the coil can increasing and then decreasing with the excitation frequency in range of $10 \mathrm{~Hz} \sim 10 \mathrm{MHz}$ [36], while when the excitation frequency is too low, the skin depth is deep; the stress we detect is a resultant stress in the range of skin depth instead of the surface stress; therefore, $0.5 \mathrm{kHz}$ is select as the excitation frequency.

To ensure the elastic deformation in the experiment, the maximal stress of the sample is required to be below the admissible stress. The admissible stress of aluminum alloy 7075 is $200 \mathrm{MPa}$ [38]. Therefore, the maximal force from the material test machine applied on the sample was calculated as $280 \mathrm{~N}$. Therefore, in the experiment, the load from the material test machine varies in the range from $0 \mathrm{~N}$ to $240 \mathrm{~N}$, with steps of $40 \mathrm{~N}$. 
TABLE 2: The geometrical and electrical parameters in the FE model.

\begin{tabular}{|c|c|c|c|c|c|}
\hline Coil & Value & Sample & Value & Air & Value \\
\hline Inner radius $(\mathrm{mm})$ & 3.5 & Height $(\mathrm{mm})$ & 6 & Height (mm) & 100 \\
\hline Outer radius (mm) & 7.5 & Width (mm) & 35 & Width (mm) & 100 \\
\hline Height $(\mathrm{mm})$ & 2 & Length $(\mathrm{mm})$ & 250 & Length (mm) & 250 \\
\hline Electrical conductivity (MS/m) & 55.85 & Electrical conductivity (MS/m) & Equation (11) & Lift-off (mm) & 0.5 \\
\hline
\end{tabular}

4.1.2. Analysis of Experimental Results. Table 1 lists the voltage signal from the Hall sensor for the different applied forces. The hall sensor SS495 in the experiment is a type of linear hall sensor. The output voltage is linearly changed with the magnetic flux density in the range of $-640 \mathrm{Gs} \sim+640 \mathrm{Gs}$. Therefore, the magnetic flux density can be calculated for different applied forces.

As can be seen in Table 1, the voltage signal and $B_{z}$ are increasing with the applied force. The potential reason is that, with the increasing applied force, the stress in the beam is linearly increasing, thereafter the resistivity of the sample is increasing and the conductivity decreases. The decreasing conductivity can result in the increasing resultant magnetic flux density, according to the principle of the eddy current technique shown in Figure 1 . The stronger the $B_{z}$, the stronger the voltage signal from hall sensor.

The relationship of $F \sim B_{z}$ is shown in Figure 6 (black full line). The expression of $B_{z} \sim F$ is fitted as Equation (9)

$$
B_{z}=0.022 F+69.79 \text {, }
$$

where the coefficient of determination $R_{s 0}$ is 0.98 .

4.2. Determination of Conductivity Distribution in the CrossSection of the Beam. For the three-point-bending simply supported beam shown in Figure 3, the stress at the point $(x, z)$ can be expressed as

$$
\sigma_{s t r}(x, z)=\frac{l}{4 I z} F \cdot z-\frac{1}{2 I z} F \cdot x \cdot z
$$

where $l$ is the length of the beam and $I_{z}$ is the inertia moment.

According to Equation (9), the electrical conductivity distribution in the three-point-bending simply supported beam can be expressed as

$$
\sigma_{\text {ele }}=\sigma_{0}+h \cdot \sigma_{0} \cdot \sigma_{\text {str }}=\sigma_{0}\left(1+\frac{l}{4 I_{z}} \mathrm{hFz}-\frac{1}{2 I_{z}} \mathrm{hFxz}\right),
$$

where $h$ is the conversion coefficient about the stress and electrical conductivity of sample, which is determined by the numerical simulation in Section 4.3.

\subsection{Determination of Coefficient $h$ by Finite Element Method}

4.3.1. Numerical Modelling by Finite Element Method. According to the symmetry in geometry and boundary conditions, a 1/4 structural model was adopted by COMSOL Multiphysics, one of the typical commercial softwares for FEM. The model was constructed according to the mesh strategy and boundary conditions investigated in [39] and shown in Figure 7. The parameters in the finite element (FE) model are listed in Table 2.

4.3.2. The Coefficient $h$. Considering the electrical conductivity distribution from Equation (11) in the FE model, the relationship curve between the applied force and the magnetic flux density in $z$-component for the different $h_{i}$ coefficients can be obtained. If $\lambda=0.01$, it will result in $h_{1}=1 / 300$ and $h_{2}=1 / 350$. The relationship curve between the applied force and the $B_{z}$ for $h=h_{1}$ and $h=h_{2}$ is shown in Figure 6 .

4.4. Applied Stress Evaluation for the Three-Point-Bending Simply Supported Beam. In three-point-bending deformation, if $B_{z}$ detected by the eddy current probe shown in Figure 5 is 72 Gauss, the applied force can then be inversely calculated as $F_{1}=97.3 \mathrm{~N}$ when $h=h_{1}$ and $F_{2}=113.3 \mathrm{~N}$ when $h=h_{2}$, as shown in Figure 6 . Therefore, the measured applied force can be expressed as $F_{m}$ as Equation (12)

$$
F m=\frac{F 1+F 2}{2}=105.3 \mathrm{~N}
$$

Furthermore, according to Figure 6, the calibration applied force $F_{c}$ is $100.3 \mathrm{~N}$ when $B_{z}=72$ Gauss. After obtaining the applied force, the stress at any point $P(x, y, z)$ in the structure can be evaluated according to Equation (10).

When the magnetic flux density $B_{z}$ detected by the hall sensor is 72 Gauss, the continuous stress distribution is calculated with Equation (10) according to the measured applied force $F_{m}=105.3 \mathrm{~N}$ in Equation (12) and shown in Figure 8(a). Figure 8(b) shows the stress distribution under the calibration applied force $F_{c}=100.3 \mathrm{~N}$ calculated by FEM. Figure 8(c) shows the absolute error of the continuous stress distribution in Figures 8(a) and 8(b).

As can be seen from Figure 8, besides the locations of the applied force and the supports, the absolute error in the whole simply supported beam is lower than $4 \mathrm{~N} / \mathrm{mm} 2$ and the relative error is lower than $8 \%$. However, the absolute error at the locations of the applied force and the supports is obvious because the stress concentration effect is taken into account in FEM, while it is not considered in the proposed method. The potential reason for the relative error of $8 \%$ in the proposed approach is the stress distribution in experiment has little difference with Equation (7), because the sample is not strictly the continuous, homogeneous, and isotropous solid.

The case study indicated that the proposed method can predict the inhomogeneous stress distribution in the simply supported beam of the metal structures by FEM according to the surface stress detected by the eddy current sensor. 


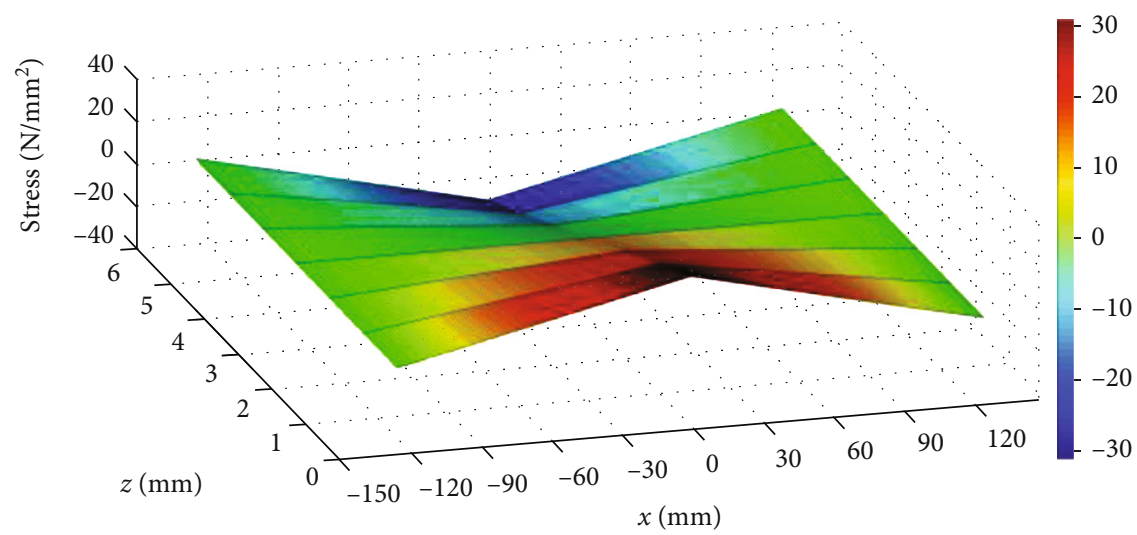

(a)

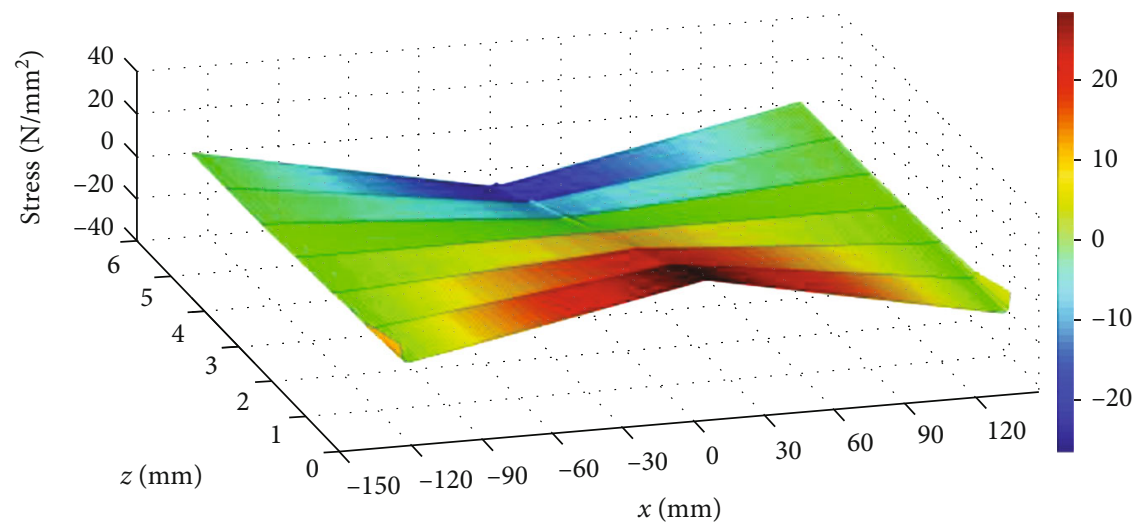

(b)

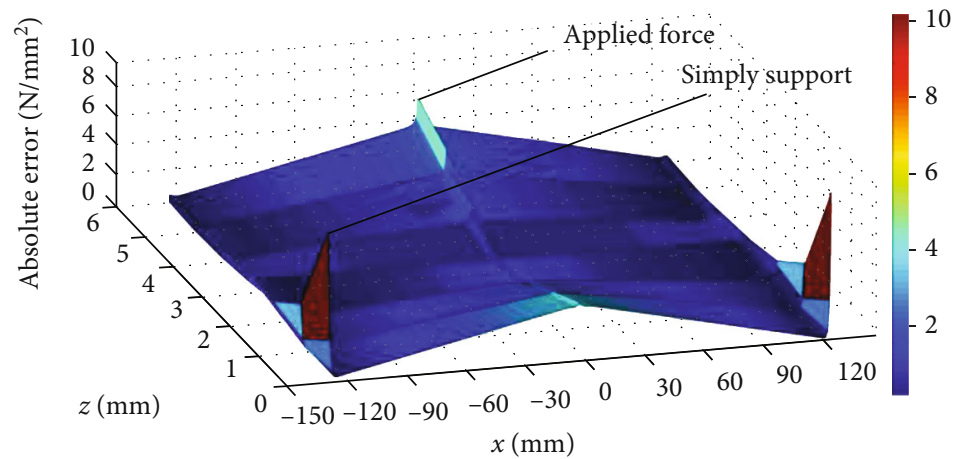

(c)

Figure 8: Continuous stress distribution (a) Stress distribution for $F_{m}=105.3 \mathrm{~N}$ calculated with Equation (10). (b) Stress distribution for $F_{c}=100.3 \mathrm{~N}$ by FEM. (c) The absolute error of the stress distribution between $F_{c}=100.3 \mathrm{~N}$ and $F_{m}=105.3 \mathrm{~N}$ when $B_{z}=72$ Gauss.

\section{Conclusions}

This paper presented a hybrid approach combining the eddy current technique and FE method, which uses the piezoresistive effect, to predict the stress distribution in early stage damages in metal structures. The surface stress can be obtained through the eddy current technique, while the FEM method can describe the relationship between surface stress and in-depth stress, so that the inhomogeneous stress can be predicted. The main conclusions are drawn below.

(1) The results of the experiment indicate that the detection signal of the eddy current technique linearly changes with the applied force on the metal structures for the bending deformation, which is consistent with the conclusion in $[28,36]$

(2) A new hybrid approach to determine the coefficient $h$ between the stress and the electrical conductivity is proposed. The coefficient $h$ bridges the gap between the magnetic flux density detected through the eddy current technique and the inhomogeneously distributed stress, which is key for inhomogeneous stress evaluation. It also provides an approach to approximate the piezoresistive coefficient of unknown material without damage 
(3) The case study shows that the proposed approach can readily measure the inhomogeneous stress distribution in bending deformation with high accuracy, which provides an example for the application of the proposed approach for inhomogeneous stress measurement under other conditions. Such as if the distribution pattern of the residual stress is known under certain conditions, this approach can be further extended to evaluate residual stress under specific working conditions for key structures, such as highspeed rail, oil/gas pipelines, airfoils, and rail and road vehicle structures

This proposed approach provides a feasible approach to predict the inhomogeneous stress or even for residual stress. However, the influence of the sample surface, material electromagnetic features, and the excitation frequency on the evaluation accuracy and the sensitivity still need to be further investigated.

\section{Data Availability}

The [DATA TYPE] data used to support the findings of this study are included within the article.

\section{Conflicts of Interest}

The authors declare that they have no conflicts of interest.

\section{Acknowledgments}

This work is supported by the National Natural Science Foundation of China (Grant No. 61960206010, Grant No. 51675087), the National Nature Science Foundation of Guangdong Province (Grant No. 2018A030313893), and also the Fundamental Research Funds for the Central Universities (Grant No. ZYGX2018J067).

\section{References}

[1] L. R. Lothhammer, M. R. Viotti, A. Albertazzi, and C. L. N. Veiga, "Residual stress measurements in steel pipes using DSPI and the hole-drilling technique," International Journal of Pressure Vessels and Piping, vol. 152, pp. 46-55, 2017.

[2] S. Taheri and A. Fatemi, "Fatigue crack behavior in power plant residual heat removal system piping including weld residual stress effects," International Journal of Fatigue, vol. 101, pp. 244-252, 2017.

[3] D. M. Spasic, S. N. Stupar, A. M. Simonovic, D. Trifkovic, and T. D. Ivanov, "The failure analysis of the star-separator of an aircraft cannon," Engineering Failure Analysis, vol. 42, pp. 74-86, 2014.

[4] W. M. Chen, L. Liu, P. Zhang, and S. R. Hu, "Non-destructive measurement of the steel cable stress based on magnetomechanical effect," Health Monitoring of Structural and Biological Systems, vol. 7650, 2010.

[5] D. K. Zhang, S. R. Ge, and Y. Qiang, "Research on the fatigue and fracture behavior due to the fretting wear of steel wire in hoisting rope," Wear, vol. 255, no. 7-12, pp. 1233-1237, 2003.
[6] K. Bobzin, W. Wietheger, M. A. Knoch et al., "Comparison of residual stress measurements conducted by X-ray stress analysis and incremental hole drilling method," Journal of Thermal Spray Technology, vol. 29, no. 6, pp. 1218-1228, 2020.

[7] Z. Wang, F. Chen, P. Li, and Y. Liu, "Research on the effect of material performances $\varepsilon \mathrm{f}$ and $\Delta \varepsilon \mathrm{c}$ on the strain fatigue life predication accuracy," International Journal of Damage Mechanics, vol. 22, no. 5, pp. 737-751, 2013.

[8] F. Y. Wang, K. M. Mao, and B. Li, "Prediction of residual stress fields from surface stress measurements," International Journal of Mechanical Sciences, vol. 140, pp. 68-82, 2018.

[9] P. J. Withers and H. K. D. H. Bhadeshia, "Residual stress. Part 1 - measurement techniques," Metal Science Journal, vol. 17, no. 4, pp. 355-365, 2001.

[10] P. Zhang, Y. D. Tan, W. X. Liu, and W. X. Chen, "Methods for optical phase retardation measurement: a review," Science China Technological Sciences, vol. 56, no. 5, pp. 1155-1164, 2013.

[11] A. Alawadi and H. Abdolvand, "Measurement and modeling of micro residual stresses in zirconium crystals in three dimension," Journal of the Mechanics and Physics of Solids, vol. 135, p. 103799, 2020.

[12] H. Kim, T. Kim, D. Morrow, and X. Jiang, "Stress measurement of a pressurized vessel using ultrasonic subsurface longitudinal wave with 1-3 composite transducers," IEEE Transactions on Ultrasonics Ferroelectrics and Frequency Control, vol. 67, pp. 158-166, 2019.

[13] H. H. Huang, J. Y. Yao, Z. W. Li, and Z. F. Liu, "Residual magnetic field variation induced by applied magnetic field and cyclic tensile stress," NDT \& E International, vol. 63, pp. 3842, 2014.

[14] J. W. Wilson, G. Y. Tian, and S. Barrans, "Residual magnetic field sensing for stress measurement," Sensors And Actuators A-Physical, vol. 135, no. 2, pp. 381-387, 2007.

[15] V. Vengrinovich, D. Vintov, A. Prudnikov, P. Podugolnikov, and V. Ryabtsev, "Magnetic Barkhausen effect in steel under biaxial strain/stress: influence on stress measurement," Journal of Nondestructive Evaluation, vol. 38, no. 2, pp. 1-8, 2019.

[16] P. Wang, X. L. Ji, X. M. Yan et al., "Investigation of temperature effect of stress detection based on Barkhausen noise," Sensors and Actuators A-Physical, vol. 194, pp. 232-239, 2013.

[17] R. B. Greene, S. Gallops, S. Funfschilling et al., "A direct comparison of non-destructive techniques for determining bridging stress distributions," Journal of the Mechanics and Physics of Solids, vol. 60, no. 8, pp. 1462-1477, 2012.

[18] Y. T. Yu and J. Guan, "Investigation of signal features of pulsed eddy current testing technique by experiments," Insight, vol. 55, no. 9, pp. 487-492, 2013.

[19] Y. T. Yu, Y. Yan, F. Wang, G. Y. Tian, and D. J. Zhang, “An approach to reduce lift-off noise in pulsed eddy current nondestructive technology," NDT \& E International, vol. 63, pp. 1-6, 2014.

[20] Q. Ma, B. Gao, G. Y. Tian, C. Yang, L. Xie, and K. Chen, "High sensitivity flexible double square winding eddy current array for surface micro-defects inspection," Sensors and Actuators A: Physical, vol. 309, p. 111844, 2020.

[21] Z. W. Liu, B. Gao, and G. Y. Tian, "Natural crack diagnosis system based on novel L-shaped electromagnetic sensing thermography," IEEE Transactions on Industrial Electronics, vol. 67, no. 11, pp. 9703-9714, 2020. 
[22] D. Desjardins, T. W. Krause, and L. Clapham, "Transient eddy current method for the characterization of magnetic permeability and conductivity," NDT \& E International, vol. 80, pp. 65-70, 2016.

[23] Y. T. Yu, T. Yang, and P. A. Du, “A new eddy current displacement measuring instrument independent of sample electromagnetic properties," NDT \& E International, vol. 48, pp. 16-22, 2012.

[24] L. M. Li, L. Q. Zhong, and X. Chen, "Residual stress caused magnetic field abnormal change upon arc welding joints," International Journal of Applied Electromagnetics and Mechanics, vol. 33, no. 3-4, pp. 1295-1301, 2010.

[25] W. Ricken, J. Liu, and W. J. Becker, "GMR and eddy current sensor in use of stress measurement," Sensors and Actuators A-Physical, vol. 91, no. 1-2, pp. 42-45, 2001.

[26] M. J. Starink and X. M. Li, "A model for the electrical conductivity of peak-aged and overaged Al-Zn-Mg-Cu alloys," Metallurgical and Materials Transactions A-Physical Metallurgy And Materials Science, vol. 34a, no. 4, pp. 899-911, 2003.

[27] M. Morozov, G. Y. Tian, and P. J. Withers, "The pulsed eddy current response to applied loading of various aluminium alloys," NDT \& E International, vol. 43, no. 6, pp. 493-500, 2010.

[28] M. Morozov, G. Y. Tian, and P. J. Withers, "Elastic and plastic strain effects on eddy current response of aluminium alloys," Nondestructive Testing and Evaluation, vol. 28, no. 4, pp. 300-312, 2013.

[29] D. Q. Zhou, M. Pan, Y. Z. He, and B. L. Du, "Stress detection and measurement in ferromagnetic metals using pulse electromagnetic method with U-shaped sensor," Measurement, vol. 105, pp. 136-145, 2017.

[30] M. P. Blodgett and P. B. Nagy, "Eddy current assessment of near-surface residual stress in shot-peened nickel-base superalloys," Journal of Nondestructive Evaluation, vol. 23, no. 3, pp. 107-123, 2004.

[31] F. Yu and P. B. Nagy, "Simple analytical approximations for eddy current profiling of the near-surface residual stress in shot-peened metals," Journal of Applied Physics, vol. 96, no. 2, pp. 1257-1266, 2004.

[32] F. Yu, M. P. Blodgett, and P. B. Nagy, "Eddy current assessment of near-surface residual stress in shot-peened inhomogeneous nickel-base superalloys," Journal of Nondestructive Evaluation, vol. 25, no. 1, pp. 17-28, 2006.

[33] B. A. Abu-Nabah, W. T. Hassan, D. Ryan, M. P. Blodgett, and P. B. Nagy, "The effect of hardness on eddy current residual stress profiling in shot-peened nickel alloys," Journal of Nondestructive Evaluation, vol. 29, no. 3, pp. 143-153, 2010.

[34] F. Yu and P. B. Nagy, "Dynamic piezoresistivity calibration for eddy current nondestructive residual stress measurements," Journal of Nondestructive Evaluation, vol. 24, no. 4, pp. 143$151,2005$.

[35] J. Ahn, E. He, L. Chen et al., "FEM prediction of welding residual stresses in fibre laser-welded AA 2024-T3 and comparison with experimental measurement," International Journal of Advanced Manufacturing Technology, vol. 95, no. 9-12, pp. 4243-4263, 2018.

[36] R. Zhu, Q. Zhang, H. Xie, X. Z. Yu, and Z. W. Liu, "Determination of residual stress distribution combining slot milling method and finite element approach," Science China Technological Sciences, vol. 61, no. 7, pp. 965-970, 2018.
[37] L. Gong, Y. Luo, X. M. Zou, and Y. He, "Influence factors of resistance for the metal material under the action of stress," in Proceedings of the 4th college physics experiment teaching seminars of China, pp. 114-117, Chongqing, China, 2006.

[38] D. X. Cheng, Mechanical design handbook, Chemical Industry Press, Beijing, China, 6th edition, 2016.

[39] Y. T. Yu, X. H. Li, A. Simm, and G. Y. Tian, "Theoretical model-based quantitative optimisation of numerical modelling for eddy current NDT," Nondestructive Testing and Evaluation, vol. 26, no. 2, pp. 129-140, 2011. 